\title{
Dynamics of Antimicrobial Peptide Encapsulation in Carbon Nanotubes: The Role of Hydroxylation
}

\begin{abstract}
Maryam Zarghami Dehaghani, ' Farrokh Yousefi, (iD ${ }^{2}$ Farzad Seidi, ' S Mohammad Sajadi, ${ }^{3,4}$

Navid Rabiee, (iD) ${ }^{5}$ Sajjad Habibzadeh, ${ }^{6}$

Amin Esmaeili,

Amin Hamed Mashhadzadeh, (iD ${ }^{8}$

Christos Spitas, 8

Ebrahim Mostafavi, iD 9,10

Mohammad Reza Saeb "

'Jiangsu Co-Innovation Center of Efficient Processing and Utilization of Forest Resources and Joint International Research Lab of Lignocellulosic Functional Materials, Nanjing Forestry University, Nanjing, 210037, People's Republic of China; ${ }^{2}$ Department of Physics,

University of Zanjan, Zanjan, 45 I95-3 I3, Iran;

${ }^{3}$ Department of Nutrition, Cihan University-

Erbil, Erbil, Iraq; ${ }^{4}$ Department of

Phytochemistry, SRC, Soran University, Soran,

Iraq; ${ }^{5}$ Department of Physics, Sharif University of

Technology, Tehran, Iran; ${ }^{6}$ Department of

Chemical Engineering, Amirkabir University of

Technology (Tehran Polytechnic), Tehran,

I591639675, Iran; ${ }^{7}$ Department of Chemical

Engineering, School of Engineering Technology and Industrial Trades, College of the North Atlantic - Qatar, Doha, Qatar; ${ }^{8}$ Mechanical and

Aerospace Engineering, School of Engineering and Digital Sciences, Nazarbayev University,

Nur-Sultan, 010000, Kazakhstan; ' Stanford

Cardiovascular Institute, Stanford University

School of Medicine, Stanford, CA 94305, USA;

${ }^{10}$ Department of Medicine, Stanford University

School of Medicine, Stanford, CA 94305, USA;

"'Department of Polymer Technology, Faculty of

Chemistry, Gdańsk University of Technology,

Gdańsk, 80-233, Poland
\end{abstract}

Correspondence: Amin Hamed

Mashhadzadeh

Mechanical and Aerospace Engineering,

School of Engineering and Digital Sciences,

Nazarbayev University, Nur-Sultan, 010000 ,

Kazakhstan

Email amin.hamed.m@gmail.com; amin.

hamedmashhadzadeh@nu.edu.kz

Ebrahim Mostafavi

Stanford Cardiovascular Institute, Stanford University School of Medicine, Biomedical Innovation Building, 240 Pasteur Drive, Palo

Alto, Stanford, CA 94304, USA

Email ebimsv@Stanford.edu; ebi.

mostafavi@gmail.com
Introduction: Carbon nanotubes (CNTs) have been widely employed as biomolecule carriers, but there is a need for further functionalization to broaden their therapeutic application in aqueous environments. A few reports have unraveled biomolecule-CNT interactions as a measure of response of the nanocarrier to drug-encapsulation dynamics.

Methods: Herein, the dynamics of encapsulation of the antimicrobial peptide HA-FD-13 (accession code 2L24) into CNTs and hydroxylated CNTs (HCNTs) is discussed.

Results: The van der Waals (vdW) interaction energy of CNT-peptide and HCNT-peptide

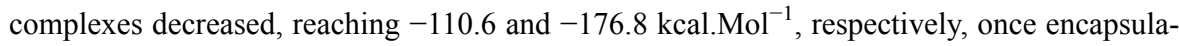
tion of the peptide inside the CNTs had been completed within $15 \mathrm{~ns}$. The free energy of the two systems decreased to -43.91 and $-69.2 \mathrm{kcal}^{-\mathrm{Mol}^{-1}}$ in the same order.

Discussion: The peptide was encased in the HCNTs comparatively more rapidly, due to the presence of both electrostatic and vdW interactions between the peptide and HCNTs. However, the peptide remained encapsulated throughout the vdW interaction in both systems. The negative values of the free energy of the two systems showed that the encapsulation process had occurred spontaneously. Of note, the lower free energy in the HCNT system suggested more stable peptide encapsulation.

Keywords: drug delivery, hydroxylation, carbon nanotubes, molecular dynamic simulation, encapsulation, antimicrobial peptide

\section{Introduction}

Even after three decades of extensive use, carbon nanotubes (CNTs) still attract worldwide attention due to their exceptional characteristics. They provide the user with large aspect ratios, ${ }^{1}$ low density, ${ }^{1}$ excellent mechanical strength, ${ }^{2,3}$ and good electrical and thermal conductivity ${ }^{4}$ and adsorption capacity. ${ }^{5} \mathrm{CNTs}$ have thus been considered for a wide range of industrial applications. ${ }^{6,7}$ For instance, today they are used in routine composite manufacturing and electronic devices. ${ }^{8,9}$ CNTs are also key nanocarriers for the delivery of drugs and therapeutic molecules. ${ }^{10}$ However, to meet a specific and targeted mission, CNTs have to be functionalized with appropriate moieties to become highly efficient for targeting, imaging, and therapeutic purposes. ${ }^{11,12}$

Due to their tubular nanostructure with single- or multiwalled features, biomolecules, such as proteins and peptides, can be encased in CNTs for sustained and targeted drug release. ${ }^{13,14}$ Moreover, CNTs are promising nanostructures for drug protection against oxidization, destructive reactions, and environmental tension ${ }^{15}$ and delaying antimicrobial-drug resistance ${ }^{16,17}$ with their unique structure that allows the penetration of cells, bacteria, and tissue. ${ }^{18}$ 
However, the hydrophobic nature of CNTs can cause aggregation, preventing biomolecular encapsulation. ${ }^{19}$ In this sense, there have been continued attempts to modify and functionalize CNTs with hydrophilic groups, such as hydroxyls, ${ }^{20}$ carboxyls, $^{21}$ and a number of hydrophilic amino acids, ${ }^{22}$ to make them water-soluble, nonimmunogenic, and less cytotoxic, which has broadened their therapeutic usage. ${ }^{23-25}$

A number of theoretical studies have been performed to evaluate the performance of functionalized CNTs as drug carriers. Maleki et $\mathrm{al}^{26}$ simulated doxorubicin encapsulation in the single-walled carboxylated CNTs (SWCNT$\mathrm{COOH}$ ) using molecular dynamic (MD) simulation. They detected van der Waals (vdW) and electrostatic (Elec) energy values of -500 and $-3,600 \mathrm{~kJ}^{-\mathrm{Mol}^{-1}}$ in neutral $\mathrm{pH}$ for SWCNT-doxorubicin and SWCNT-COOH-doxorubicin, respectively. However, the carboxyl groups showed no charge in an acidic environment, where vdW interaction was dominant in the adsorption process, facilitating release of the drug in cancerous tissue. Moradnia et $\mathrm{al}^{27}$ reported adsorption of gemcitabine on the surface of polyethylene glycol (PEG)-modified SWCNTs using density functional theory. It was understood that $\mathrm{OH}$ hydrogen bonds between the PEG and gemcitabine dominated the intermolecular interactions in the drug-SWCNT complex. Kamel et $\mathrm{al}^{28}$ demonstrated the role of hydrogen bonding between carboxyl groups of functionalized CNTs and the anticancer drug flutamide in stabilization of conformation of the drug-CNT complex. Moreover, the vdW interaction in carboxylated CNT-drug complex containing CNTs with chirality of $(10,5)$ showed a more negative value $(-350 \mathrm{~kJ}$. $\mathrm{Mol}^{-1}$ ) than systems in which CNTs with chirality of $(10,0)$ and $(10,10)$ were considered $(-100$ and $-150 \mathrm{~kJ}$. $\mathrm{Mol}^{-1}$, respectively). Dehneshin et al ${ }^{29}$ used valine and phenylalanine moieties to functionalize CNTs and simulate the adsorption of various drugs, including sunitinib, streptozotocin, and sorafenib using MD simulation. Although adsorption of sorafenib and sunitinib on functionalized CNTs occurred through $\pi-\pi$ stacking and hydrogen bonding, streptozotocin absorbed on CNTs via hydrogen bonding. Hashemzadeh et $\mathrm{al}^{30}$ simulated the adsorption of paclitaxel on the surface of CNTs and PEG-functionalized-CNTs, wherein functionalized CNTs enhanced the solubility of the CNTs in aqueous solution and remained

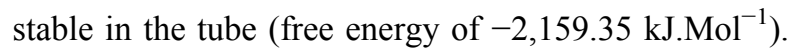

In light of these examples, it can be concluded that choosing an appropriate functional group is key to understanding and keeping stable the encapsulation of drugs in
CNTs. MD simulation appears to be successful in capturing mechanical and thermal properties of nanostructures,31-38 and more importantly the interaction of drugs with nanostructures. ${ }^{39-42}$ CNT-based drug-delivery systems can be simulated in terms of variation in vdW and Elec energies. The possibility of encapsulation of the 13amino-acid peptide HA-FD-13 (ID 2L24) in the CNT and hydroxylated CNT (HCNTs) and stability of the drug-CNT complex was simulated in the present study. This drug is an $\alpha-$ helical antimicrobial peptide derived from the fusion domain (FD) of hemagglutinin (HA) of the influenza virus, and has antimicrobial characteristics, penetrating the anionic microbial membranes. ${ }^{43} \mathrm{vdW}$ and Elec interactions between drug and NTs, as well as the potential of mean force (PMF) of the encapsulated peptide, were calculated using MD simulation and compared for both unmodified and functionalized CNTs.

\section{Simulation Method}

In the present study, investigation of the insertion process of the $\alpha$-helical antimicrobial peptide HA-FD-13 into the CNT and HCNT and subsequently the stability of the encapsulated HA-FD-13 in NTs was performed through MD calculation using LAMMPS software. ${ }^{44}$ HA-FD-13 (containing amino acids Ile,Phe,Gly,Ala,Lys,Asn,Trp, and $\mathrm{NH}_{2}$ ) was chosen from the Protein Data Bank (https:// www.rcsb.org/structure/2L24). This antimicrobial peptide has a positive charge so that it can penetrate anionic microbial membranes.

VMD software was used to visualize the simulated encapsulation process. ${ }^{45}$ Considering the size of HA-FD13, a CNT and HCNT with chirality of armchair $(20,20)$ and length of $40 \AA$ were selected as nanovehicles for this peptide. The HCNT contained hydroxyl groups on the edge with a degree of functionalization of $2.82 \%$, which was calculated thus:

$\begin{aligned} & \text { Degree of functionalization }= \begin{array}{l}\text { Number of hydroxylated } \\ \text { carbon atoms in } \mathrm{CNT}-\mathrm{OH}\end{array} \\ & \begin{array}{l}\text { Total number of carbon } \\ \text { atoms inCNT }-\mathrm{OH}\end{array} \\ & \times 100\end{aligned}$

At the beginning of the simulation, HA-FD-13 was situated at an initial distance of $2 \AA$ from the NT. The axial direction of the NT was parallel to the $z$-axis of the simulation box. CNT-HA-FD-13 and HCNT-HA-FD-13 complexes were immersed in simulation boxes containing 
TIP3P water molecules, as well as counterions to neutralize the simulated solution with periodic boundary conditions. All MD simulations were performed applying a CHARMM27 force field. ${ }^{46}$ Tersoff potential was used to consider interactions among carbon atoms of NTs. ${ }^{47}$ To verify the encapsulation process of the peptide, in the first step minimization of the system was achieved in the NVT ensemble at $300 \mathrm{~K}$ while the NT was fixed. In the next step, the MD runs were performed in the NPT ensemble for $15 \mathrm{~ns}$ with a time step of 1 fs. The inner and outer cutoff distances for the Lennard-Jones and coulombic potential were considered to be 8 and $12 \AA$, respectively. The parameters of Lennard-Jones potential for cross-vdW interactions between unbonded atoms was estimated using the Lorentz-Berthelot combination rule. ${ }^{48}$ The vdW interaction between HA-FD-13 and the CNT was calculated: ${ }^{45}$

$$
E_{v d W-i n t}(t)=E_{\text {peptide }+C N T}(t)-\mathrm{E}_{\text {peptide }}(t)-E_{C N T}(t)
$$

where $E_{v d W}$ refers to vdW energy between HA-FD-13 and the NT and $E_{\text {peptide }+C N T} \mathrm{vdW}$ interaction of HA-FD-13 combined with the CNT. $E_{\text {peptide }}$ and $E_{C N T}$ stand for vdW energies of HA-FD-13 and the CNT, respectively. To evaluate the stability of the peptide encapsulated inside the CNT, an external force was loaded on HA-FD-13 along the $z$-axis of the NT to pull it out in the direction opposite to the penetration process. The spring constant $\mathrm{k}$ and pull-


$0.005 \AA$. . s $^{-1}$, respectively. ${ }^{49}$ The pulling process was simulated ten times to compute the PMF profile using Jarzynski's equality: ${ }^{46}$

$$
e^{-\beta \Delta G}=<e^{-\beta W}>
$$

where $\Delta \mathrm{G}$ and $\mathrm{W}$ correspond to the free energy discrepancy between two states and the work performed on the system, respectively. $\beta$ is equal to $\left(K_{B} \mathrm{~T}\right)^{-1}$, where $K_{B}$ stands for the Boltzmann constant. The temperature and pressure of the system were adjusted to about $300 \mathrm{~K}$ and $101.3 \mathrm{kPa}$ through applying Langevin dynamics and the Langevin piston Nosé-Hoover method, respectively. ${ }^{50}$

\section{Results and Discussion}

\section{Localization of HA-FD-I 3 Within the CNT-Peptide and HCNT-Peptide Complexes}

The encapsulation process of HA-FD-13 inside the CNT and HCNT was investigated through MD simulation. Snapshots of these processes were taken by VMD to demonstrate the positions of the peptide at different times in the simulation box. Figures 1 and 2 show these snapshots relating to the encapsulation of the peptide into the CNT and HCNT, respectively. As can be observed, HA-FD-13 was successfully encapsulated in the cavity of both the CNT and HCNT; however, the insertion process of the peptide confinement accelerated in the case of the HCNT, which means that the peptide overcame the energy barrier in the vicinity of the NT more rapidly. Moreover, the peptide remained stably encapsulated throughout the $\mathrm{vdW}$ interaction between the conjugated aromatic rings of the peptide and the interior walls of the NT to the end of the simulation (15 ns) in both systems. Conformational changes taking place inside the NTs are provided in Supplementary Information (Video 1: [CNT-peptide] and Video 2: [HCNT-peptide]). The center of mass (CoM) distance between the peptide and the CNT and HCNT are depicted in Figure $3 \mathrm{~A}$ and $\mathrm{B}$, respectively. The sudden decrease in CoM distance at the beginning of the simulation in both systems demonstrated that the peptide was self-adjusting before entering the cavity of the NTs. The same downward trend was observed in work done by Kang et al. ${ }^{49}$ They reported that the protein SmtA showed self-adjusted conformational changes before entering CNTs.

After the sudden drop had occurred in this curve for the CNT-peptide complex, it reached a minimum of $3.62 \AA$ before fluctuating around the specified value at $2 \mathrm{~ns}$, followed by the second minimum of $4.3 \AA$ at $8 \mathrm{~ns}$. On the other hand, for the HCNT-peptide complex, the CoM distance showed fluctuation around $7.5 \AA$ after the initial drop, with no sign of a minimum. It can be posited that the presence of the hydroxyl groups in the entrance edge of the CNT created hydrogen bonding between the CNT and the peptide and subsequently a coulombic interaction (as shown in Figure 3E) such that the peptide was rapidly adsorbed into the NT. This caused the sudden drop in CoM distance and the following fluctuation. In other words, the adsorption interaction between the $\mathrm{OH}$ groups on the edge and the peptide did not allow the peptide to reach the center of the NT and caused more intense fluctuation compared to the pristine CNT. For the CNT-peptide complex, the revealed two minima in the CoMdistance curve showed that after insertion of the peptide in the NT, it could more easily pass the edge of the NT and experience a sudden change in CoM distance with smooth fluctuating changes between these minima, due to the absence of the coulombic interaction between the CNT 


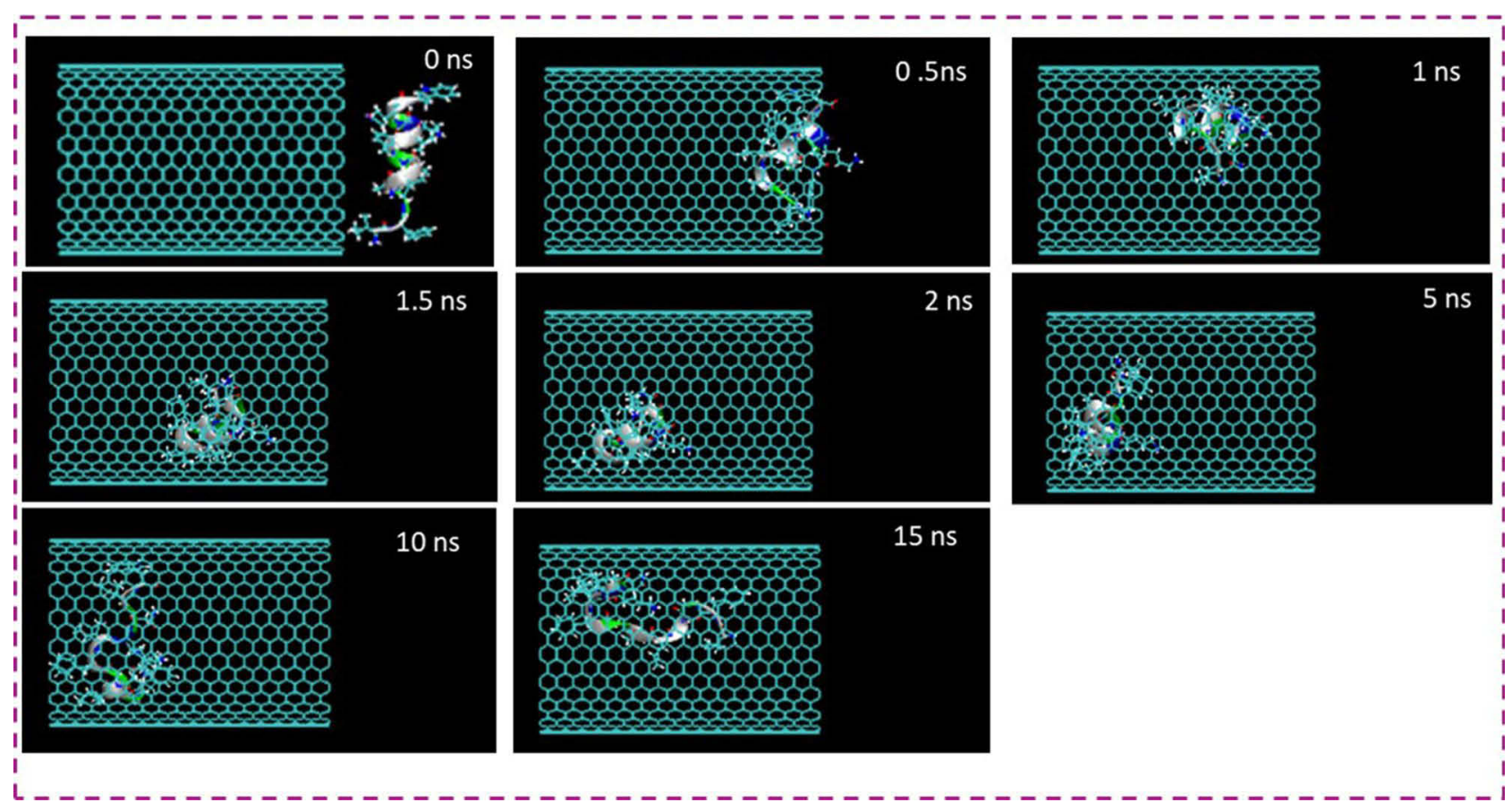

Figure I Representative snapshots of insertion of the HA-FD-13 peptide into an armchair $(20,20)$ CNT at various times. For clarity, molecules of water have not been shown.

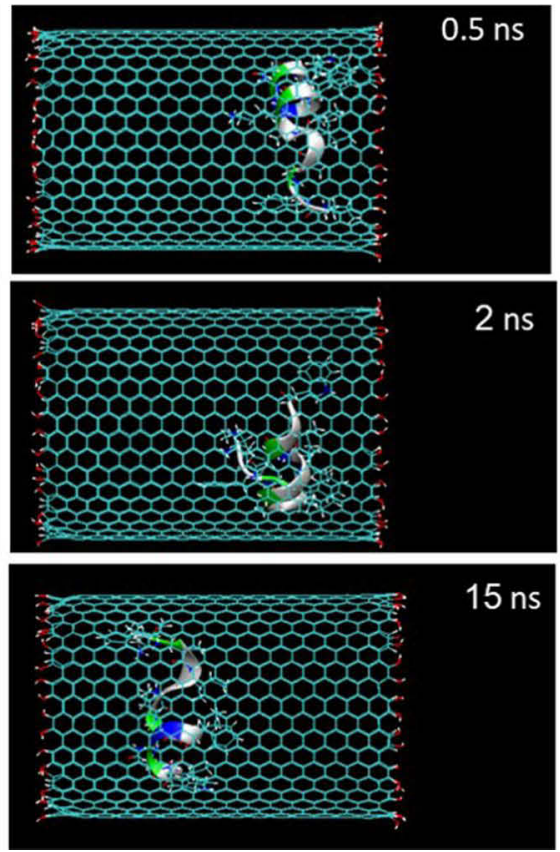

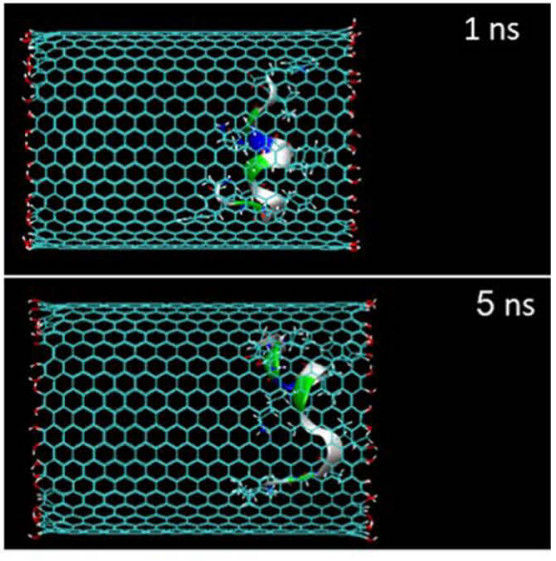

5 ns

Figure 2 Representative snapshots of insertion of HA-FD-I3 into an armchair $(20,20)$ HCNT at various times. For clarity, molecules of water have not been shown. and peptide that caused that the vdW interactions being the dominant parameter in determining the encapsulation process.
Variations in vdW interaction energy between the peptide and NT in the CNT-peptide and HCNT-peptide complexes with simulation time during the encapsulation 



Figure 3 Center of mass (CoM) distance between (A) HA-FD-I3 and the CNT; (B) HA-FD-I3 and the HCNTs as a function of simulation time; vdW interaction between (C) HA-FD-I3 and the CNT (20,20); (D) HA-FD-I3 and the HCNT $(20,20)$; (E) coulombic interaction between HA-FD-I3 and the HCNT (20,20) as a function of simulation time.

process are shown in Figure $3 \mathrm{C}$ and $\mathrm{D}$, respectively. As expected, vdW interaction energies of the CNT-peptide and HCNT-peptide complexes diminished with the drop in CoM distance between peptide and NT during the encapsulation process, reaching -110.6 and $-176.8 \mathrm{kcal}$. $\mathrm{Mol}^{-1}$, respectively after the complete adsorption of the peptide into the NT cavity at $15 \mathrm{~ns}$. The vdW interaction energy of the system containing the modified CNT decreased, which showed that the stronger vdW interaction between the HCNT and the peptide resulted in the development of a more favourable drug-delivery system with the ability to penetrate cells. ${ }^{51}$ A similar decreasing trend in the vdW energy of insertion of DNA oligonucleotides and collagen-like peptides into CNTs has been observed in research performed by Gao et $\mathrm{al}^{52}$ and Kang et $\mathrm{al}^{53}$ respectively. In another work done by Maleki et al, ${ }^{54}$ Elec and $\mathrm{vdW}$ interactions between doxorubicin and CNTs were determined. According to their results, as a result of the zero value of Elec interactions, the absorbance of doxorubicin in the CNTs was due to vdW interactions with favorable negative values. Hashemzadeh et $\mathrm{al}^{30}$ showed that $\mathrm{vdW}$ interactions between the anticancer drug paclitaxel and CNTs modified with PEG were stronger, with lower negative values than the vdW interaction 

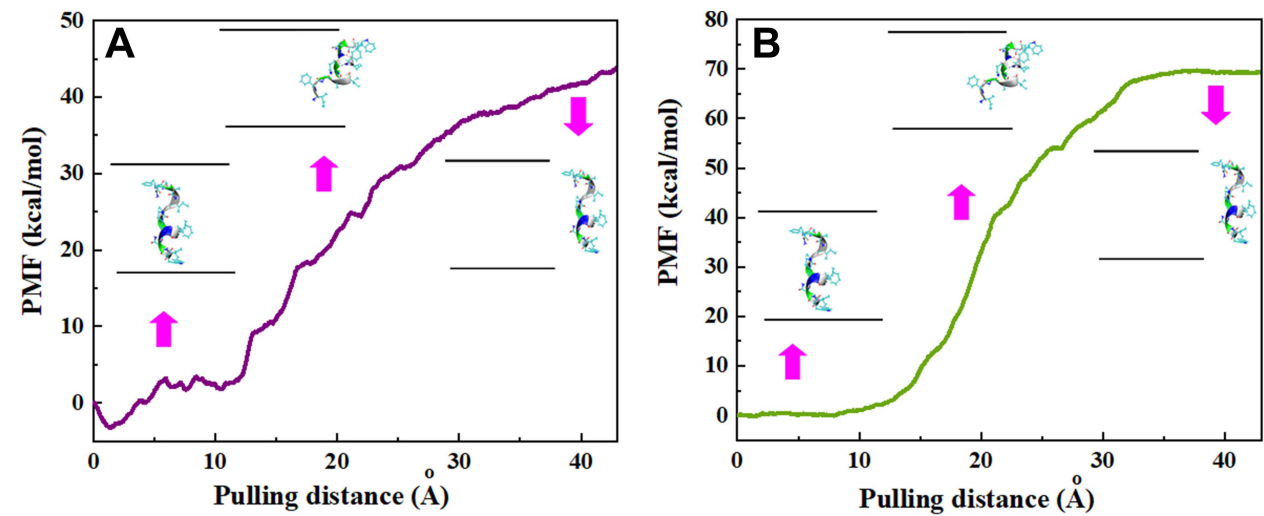

Figure 4 Potential of mean force (PMF) for systems of (A) CNT-peptide and (B) HCNT-peptide complexes computed from ten pullings through the MD simulation. The images represent the positions of HA-FD-13 corresponding to the z-coordinate along the (A) CNT and (B) HCNT at key positions.

of the pristine CNT-drug complex. As another observation from Figure $3 \mathrm{C}$, the decreasing trend of $\mathrm{vdW}$ interaction energy of the CNT-peptide complex contained two steps: first at the beginning of the simulation, and second starting at $6 \mathrm{~ns}$ and ending at $9 \mathrm{~ns}$ of the simulation. This indicates that the peptide did not undergo conformational changes outside these periods. In the second decrease, the conformation of the peptide varied such that the vdW interaction energy reached the lowest negative value.

\section{Free Energy Calculation by MD Simulation}

At the end of the simulation, when the peptide had completely adsorbed inside the NTs at $15 \mathrm{~ns}$, its PMF profiles were calculated by pulling it out through the MD simulation at the speed of $0.005 \AA . \mathrm{ps}^{-1,}$ which was selected based on the speed of the insertion process. This simulation was performed five times to obtain the average value of work (W) at each pulling distance, which is shown as PMF profiles in Figure 4A and B for the CNT and HCNT systems, respectively. Peptide positions along the $z$-axis corresponding to the NTs are depicted also. As can be observed, the free energies of the simulated pristine-CNT and HCNT systems were elevated during the pulling process and reached 43.91 and $69.2 \mathrm{kcal}^{-\mathrm{Mol}^{-1}}$, respectively, at a pulling distance of $43 \AA$. These results indicated that the encapsulation process of the peptide inside the CNT and HCNT occurred spontaneously, with free energy of -43.91 and $-69.2 \mathrm{kcal}^{\mathrm{M} \mathrm{Mol}^{-1}}$, respectively. However, the lower free energy in the HCNT system showed more stability of the encapsulated peptide inside the cavity of the NT than the pristine CNT system. A similar spontaneous process was observed in work done by Veclani et al. ${ }^{55}$ They obtained free energy value of $-9.5 \mathrm{kcal}$. $\mathrm{Mol}^{-1}$ for the encapsulation process of ciprofloxacin on the surface of CNTs. Ghadri et a ${ }^{56}$ reported that enhancement of active sites on CNTs caused an increase in hydrogen bonding between the drug fulvestrant and functional groups of CNTs such that the stronger affinity of the drug with the functionalized CNTs caused a lower free energy $\left(-373.38 \mathrm{kcal}^{\mathrm{M} \mathrm{Mol}^{-1}}\right)$ than the system with pristine CNTs.

The energy contributions of the whole system in the spontaneous encapsulation of HA-FD-13 inside the CNT

Table I Energy contributions (kcal.Mol ${ }^{-1}$ ) in the spontaneous encapsulation of HA-FD-I3 inside CNTs $(20,20)$. $\Delta G$ : Free energy variation of the whole system in the encapsulation process. $\Delta S$ : Entropy variation of the whole system. $\Delta H$ : Enthalpy variation of the whole system. $\Delta E_{k}$ : Kinetic energy variation of the system in the encapsulation process. $V(\Delta P)$ : Multiplication of the volume of the system and pressure change of the whole system, $\Delta E_{p}$ : Potential energy variation of the system, composed of vdW energy change $\left(\Delta E_{v d W}\right)$, Elec energy change $\left(\Delta E_{\text {ele }}\right)$, and bonded-atom energy change $\left(\Delta E_{\text {bonded }}\right)$

\begin{tabular}{|l|c|c|c|c|c|}
\hline \multicolumn{5}{|c|}{$\Delta G-43.91$} \\
\hline \multirow{3}{*}{$-T \Delta S 2,949.62$} & \multicolumn{4}{|c|}{$\Delta H-2,993.53$} \\
\cline { 2 - 6 } & $V(\Delta P)-2,576.543$ & $\Delta E_{k}$ II.273 & & \multicolumn{4}{|c|}{$\Delta E_{p}-428.26$} \\
\cline { 2 - 6 } & & & $\Delta E_{\text {vdW }}-310.5$ & $\Delta E_{\text {ele }}-198.28$ & $\Delta E_{\text {bonded }} 80.52$ \\
\hline
\end{tabular}


Table 2 Energy contributions (kcal.Mol ${ }^{-1}$ ) in the spontaneous encapsulation of HA-FD-I3 inside the HCNT $(20,20)$. $\Delta G$ : Free energy variation of the whole system in the encapsulation process. $\Delta S$ : Entropy variation of the whole system. $\Delta H$ : Enthalpy variation of the whole system. $\Delta E_{k}$ : Kinetic energy variation of the system in the encapsulation process. $V(\Delta P)$ : Multiplication of the volume of the system and the pressure change of the whole system. $\Delta E_{p}$ : Potential energy variation of the system, composed of vdW energy change $\left(\Delta E_{v d W}\right)$, Elec energy change $\left(\Delta E_{\text {ele }}\right)$, and bonded-atom energy change $\left(\Delta E_{\text {bonded }}\right)$

\begin{tabular}{|c|c|c|c|c|c|}
\hline \multicolumn{6}{|c|}{$\Delta G-69.2$} \\
\hline \multirow[t]{3}{*}{$-T \Delta S I, 648.42$} & \multicolumn{5}{|c|}{$\Delta H-I, 7 \mid 7.62$} \\
\hline & \multirow[t]{2}{*}{$V(\Delta P)-|, 257.7|$} & \multirow[t]{2}{*}{$\Delta E_{k} \mid 80.96$} & \multicolumn{3}{|c|}{$\Delta E_{p}-640.87$} \\
\hline & & & $\Delta E_{v d W}-7.176$ & $\Delta E_{\text {ele }}-677.46$ & $\Delta E_{\text {bonded }} 43.766$ \\
\hline
\end{tabular}

and HCNT are given in Tables 1 and 2, respectively. Considering the definition of free energy $(\Delta \mathrm{G}=\Delta \mathrm{H}-$ $\mathrm{T} \Delta \mathrm{S}$, where $\Delta \mathrm{H}$ and $\Delta \mathrm{S}$ are changes in enthalpy and entropy, respectively), it can be concluded that the encapsulation process was an enthalpy-driven phenomenon, due to the greater contribution of enthalpy change to the free energy of the whole system and decrease in entropy during the encapsulation process in both systems. ${ }^{57}$ As shown in Tables 1 and 2, the potential energy of the whole system $(\Delta \mathrm{P})$ as a part of enthalpy change consists in variations in $\operatorname{vdW}$ energy $\left(\Delta \mathrm{E}_{\mathrm{vdW}}\right)$, Elec energy $\left(\Delta \mathrm{E}_{\text {ele }}\right)$, and bondedatom energy $\left(\Delta \mathrm{E}_{\text {bonded }}\right)$. For the pristine $\mathrm{CNT}$ system, the greater drop in vdW energy (310.5 kcal. $\left.\mathrm{Mol}^{-1}\right)$ compared to the decrease in Elec energy (198.28 kcal.Mol ${ }^{-1}$ ) proved that the vdW interaction played a more important role in the encapsulation process. In the HCNT system, the reduction in Elec energy ( $-677.48 \mathrm{kcal}^{\mathrm{M} \mathrm{Mol}^{-1}}$ ) was found to be the more important contributor to the process than the slight decrease in vdW energy $\left(-7.176 \mathrm{kcal}^{\mathrm{M}} \mathrm{Mol}^{-1}\right)$. The increase in $\mathrm{E}_{\text {bonded }}$ in both systems (80.52 and $43.766 \mathrm{kcal}$. $\mathrm{Mol}^{-1}$ for CNT and HCNT, respectively) corresponded to changes in energy of bonded atoms and subsequently conformational changes in the systems.

\section{Variations in Conformation of HA-FD-I 3} Changes in the conformation of HA-FD-13 in the CNT and HCNT systems at 0 and $15 \mathrm{~ns}$ of the MD simulation are illustrated in Figures 5A and 6A, respectively. The left images in these figures correspond to the peptide immersed in an aqueous solution in its natural structure at $0 \mathrm{~ns}$. The right images show the state in which the conformation of the peptide was adjusted to the interior geometry of the NTs at 15 ns. Similar alterations in the arrangement and conformation of doxorubicin encapsulated within CNTs in accordance with NT chirality and diameter was reported by Zhang et al. ${ }^{58}$ Root-mean-square deviations (RMSDs) revealing the variations in the peptide conformation in both systems as a function of simulation time are shown in Figures $5 \mathrm{~B}$ and $6 \mathrm{~B}$, respectively. As can be observed, for both systems, the RMSD showed fluctuation of 6-16 from the beginning of the simulation to $12 \mathrm{~ns}$, which indicates the continuous conformational alteration of the peptide, or in other words self-adjustment of the peptide according to the interior geometry of the NT. However, the conformation of the peptide in the HCNTpeptide complex varied more intensely than the conformational changes in the CNT-peptide complex at the beginning of the simulation. This shows that the hydrogen bonding creating the Elec interaction between the hydroxyl groups on the edge of the CNT and the peptide caused not only the rapid adsorption of the peptide but also intense conformational change of the peptide.

After 12 ns of the simulation, the peptide reached a more stable state, with less pronounced changes in conformation and subsequent RMSD so that the aromatic rings were situated parallel to the interior wall of the NT, causing the strong vdW interaction. This observation is in good agreement with the $\mathrm{vdW}$ interaction energies obtained between the peptide and both NTs. A similar structural variation during the encapsulation of the ssDNA oligomer inside the CNT was observed by Shen et al. ${ }^{59}$ They showed that the RMSD of the biomolecule fluctuated slightly after complete encapsulation, and its conformation caused the creation of a $\pi-\pi$ stacking interaction. In their other work, ${ }^{60}$ it was reported that changes in RMSD for the encapsulated biomolecule were due to differences in the affinity of its hydrophobic and hydrophilic parts with the solvent and the NT, respectively. Besides the calculation of the RMSD, determining alterations in gyration radius of HA-FD-13 during the insertion 

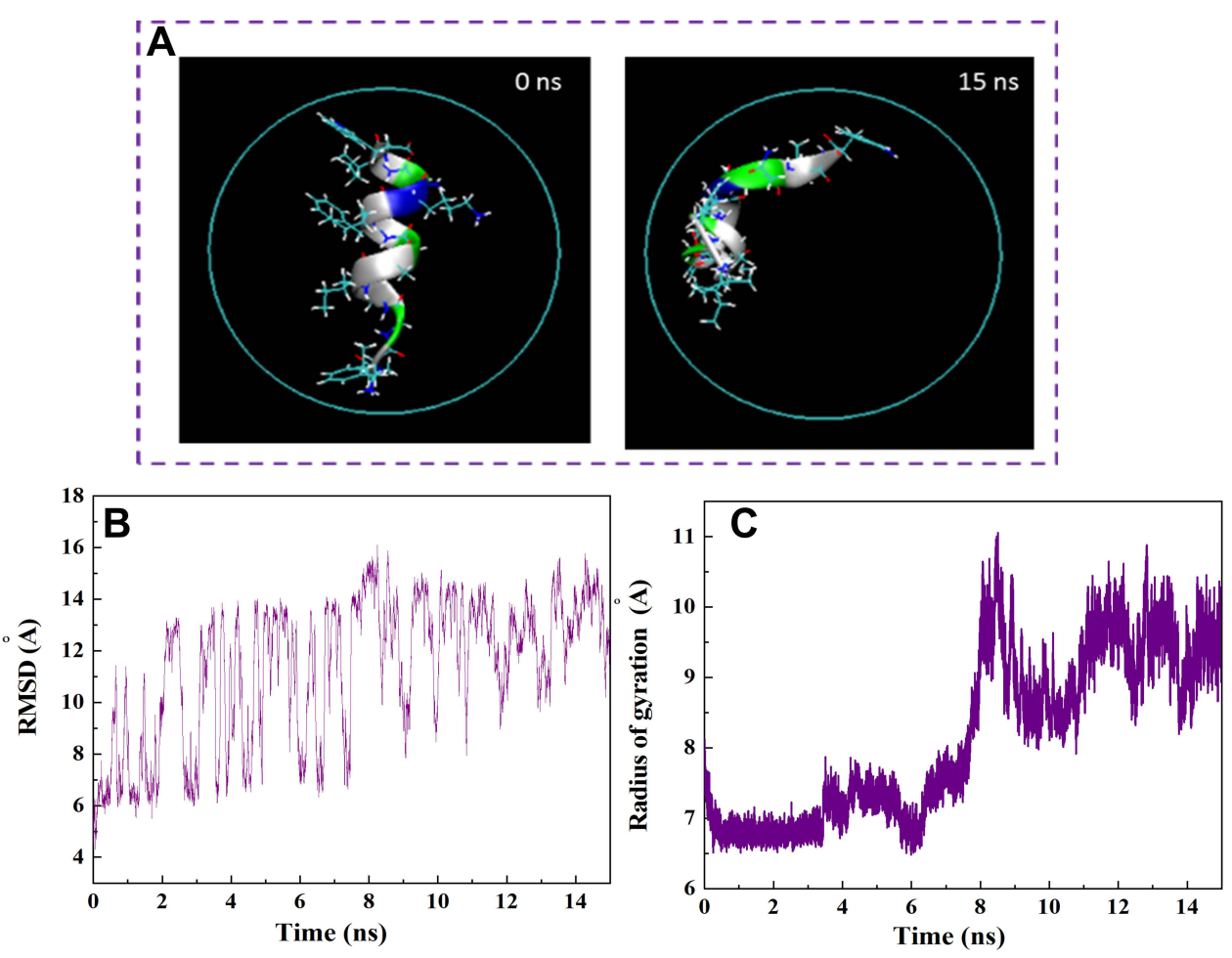

Figure 5 (A) Axial views of HA-FD-13 at 0 and 15 ns in the MD simulation. For the sake of clarity, molecules of water have not been shown. (B) Root mean square deviation (RMSD) of HA-FD-I3 as a function of simulation time. (C) Radius gyration of HA-FD-I3 as a function of simulation time in the CNT- HA-FD-I3 complex.

process provided beneficial details about the conformational changes of the peptide. Figures $5 \mathrm{C}$ and $6 \mathrm{C}$ show these variations in the CNT and HCNT systems, respectively, during the simulation time. In both systems, the structure of peptides stretched to adjust to NT geometry through peptide-NT vdW interactions. This conformational change was associated with the higher gyration radius of the confined peptide than the unconfined peptide.

Variations in distances between the CoM of HA-FD-13 and the central axis of the CNT and HCNT as a function of simulation time are demonstrated in Figure $7 \mathrm{~A}$ and $\mathrm{B}$, respectively. It can be seen that the peptide was in a position in the simulation box such that that the CoM of the peptide was situated on the central axis of the CNT and HCNT at the beginning of the simulation. After the simulation had started, the peptide moved toward one side of the CNT and HCNT, causing an immediate increase in peptide-axis distance. These sudden movements correlated with the vdW interaction between the wall of the CNT and the peptide and both vdW and Elec interactions between the peptide and HCNT. However, these conformations were not stable and varied through the adsorption of the peptide to the other sidewall of the NTs, revealing a sudden drop in peptide-axis distance. After total insertion of the peptide in NTs, the distance between the CoM of the peptide and the central axis of the NTs fluctuated continuously, due to the completion of adsorption of the hydrophilic and hydrophobic groups of the protein by the water molecules andNTs, respectively. The slight fluctuation in the curve of peptide-axis distance in the HCNT system was related to the greater stability of the encapsulated peptide in HCNT than the pristine CNT.

Figure $7 \mathrm{C}$ and $\mathrm{D}$ present the changes in potential energy of HA-FD-13 during encapsulation into the CNT and HCNT, respectively. For the peptide in the HCNT, it was observed that its potential energy dwindled during the first $0.5 \mathrm{~ns}$ of the insertion procedure to $-50 \mathrm{kcal}^{\mathrm{Mol}}{ }^{-1}$, favoring the rapid adsorption and stability of the peptide in the cavity of the NT under the effect of both vdW and Elec interactions between the peptide and NT. However, for the peptide in the CNT-peptide complex, an unfavourable level of potential energy (100 kcal.Mol $\left.{ }^{-1}\right)$ correlated with less stability for the encapsulated peptide. ${ }^{49}$

\section{Conclusion}

In this research, the encapsulation process of the antimicrobial peptide HA-FD-13 inside a CNT and HCNT in an 

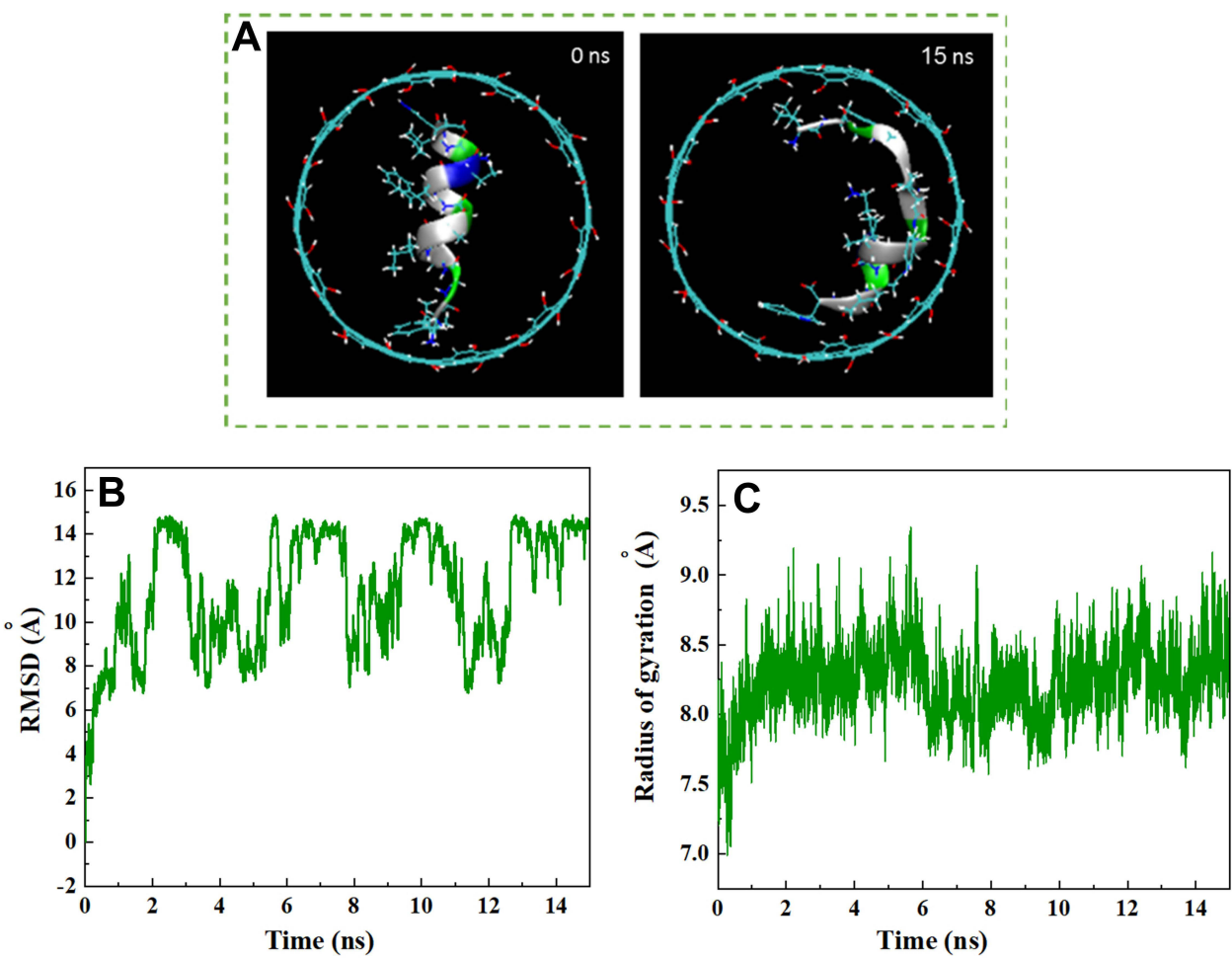

Figure 6 (A) Axial views of HA-FD-13 at 0 and 15 ns in the MD simulation. For the sake of clarity, molecules of water have not been shown. (B) Root mean square deviation (RMSD) of HA-FD-I3 as a function of simulation time. (C) Radius gyration of HA-FD-I3 as a function of simulation time in the HCNT-peptide complex.
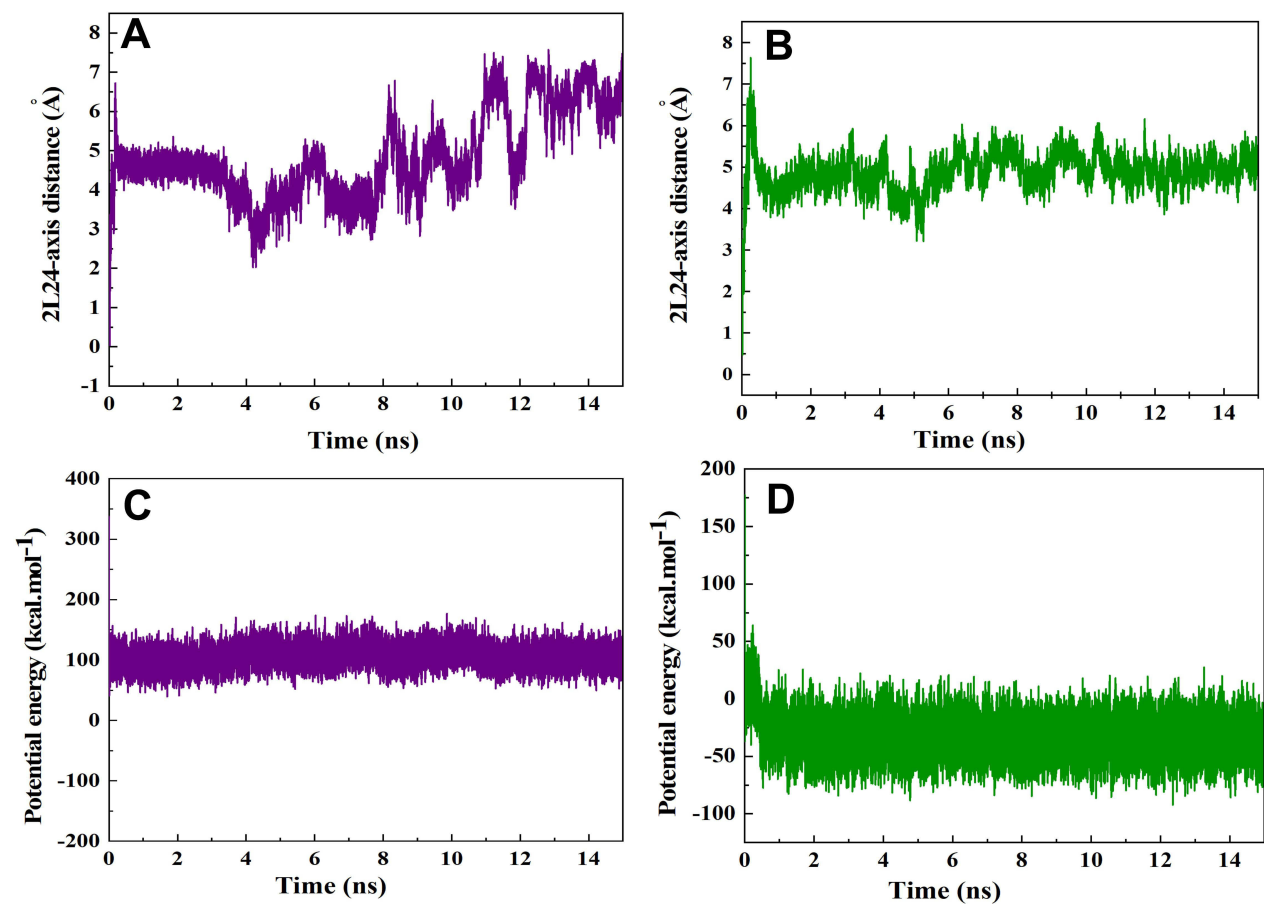

Figure 7 Distance between the center of mass (CoM) of HA-FD-I3 and the central axis of (A) the CNT and (B) HCNT as a function of simulation time. Potential energy of HA-FD-I 3 in the (C) CNT-peptide complex and (D) HCNT-peptide as a function of simulation time. 
aqueous solution was compared through MD simulation. The most important results of our research are:

1. The peptide was encapsulated in the cavity of HCNT more rapidly due to the presence of both Elec and vdW interactions between the peptide and NT.

2. The peptide remained encapsulated throughout the $\mathrm{vdW}$ interaction between the conjugated aromatic rings of the peptide and the interior walls of the NT until the end of the simulation ( $15 \mathrm{~ns}$ ) in both systems.

3. The sudden decrease in CoM distance between the peptide and NTs at the beginning of the simulation in both systems demonstrated self-adjustment of the peptide before entering the cavity of NTs.

4. The vdW interaction energies of the CNT-peptide and HCNT-peptide complexes diminished, reaching

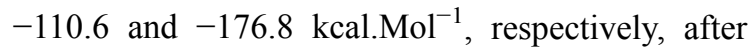
complete adsorption of the peptide into the NT cavity at $15 \mathrm{~ns}$.

5. Encapsulation of the peptide inside the CNT and HCNT occurred spontaneously, with free energy of

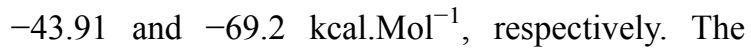
lower free energy in the HCNT system showed more stability for the encapsulated peptide.

6. RMSD results showed that the conformation of the peptide in the HCNT-peptide complex varied more intensely at the beginning of the simulation. After 12 $\mathrm{ns}$, in both systems the peptide had reached a more stable state, with lower changes in conformation and subsequent RMSD such that the aromatic rings of the peptide were placed parallel to the interior wall of the NTs, causing the strong vdW interaction.

7. The potential energy of the peptide reached -50 and $100 \mathrm{kcal}^{\mathrm{Mol}}{ }^{-1}$ in the CNT and HCNT systems, respectively, revealing the greater stability of the HCNT complex.

\section{Acknowledgements}

Ebrahim Mostafavi would like to acknowledge the support from the National Institute of Biomedical Imaging and Bioengineering (5T32EB009035).

\section{Author Contributions}

All authors made a significant contribution to the work reported, whether in conception, study design, execution, acquisition of data, analysis, and interpretation, or in all these areas, took part in drafting, revising, or critically reviewing the article, gave final approval to the version to be published, have agreed on the journal to which the article has been submitted, and agree to be accountable for all aspects of the work.

\section{Funding}

This work was funded by Nazarbayev University grant OPCRP2020002.

\section{Disclosure}

The authors declare no conflicts of interest.

\section{References}

1. Ibrahim KS. Carbon nanotubes-properties and applications: a review. Carbon Lett. 2013;14:131-144.

2. Mashhadzadeh AH, Fereidoon A, Ahangari MG. Surface modification of carbon nanotubes using 3-aminopropyltriethoxysilane to improve mechanical properties of nanocomposite based polymer matrix: experimental and density functional theory study. Appl Surf Sci. 2017;420:167-179. doi:10.1016/j.apsusc.2017.05.148

3. Fereidoon A, Mashhadzadeh AH, Rostamiyan Y. Experimental, modeling and optimization study on the mechanical properties of epoxy/highimpact polystyrene/multi-walled carbon nanotube ternary nanocomposite using artificial neural network and genetic algorithm. Sci Eng Compos Mater. 2013;20(3):265-276. doi:10.1515/secm-2012-0178

4. Rostamiyan Y, Mohammadi V, Mashhadzadeh AH. Mechanical, electronic and stability properties of multi-walled beryllium oxide nanotubes and nanopeapods: a density functional theory study. $J \mathrm{Mol}$ Model. 2020;26(4):1-13. doi:10.1007/s00894-020-4328-5

5. Mashhadzadeh AH, Fathalian M, Ghorbanzadeh Ahangari M, et al. DFT study of $\mathrm{Ni}, \mathrm{Cu}, \mathrm{Cd}$ and $\mathrm{Ag}$ heavy metal atom adsorption onto the surface of the zinc-oxide nanotube and zinc-oxide graphene-like structure. Mater Chem Phys. 2018;220:366-373. doi:10.1016/j. matchemphys.2018.09.016

6. Gupta N, Gupta SM, Sharma SK. Carbon nanotubes: synthesis, properties and engineering applications. Carbon Lett. 2019;29 (5):419-447. doi:10.1007/s42823-019-00068-2

7. Venkataraman A, Amadi EV, Chen Y, et al. Carbon nanotube assembly and integration for applications. Nanoscale Res Lett. 2019;14 (1):220. doi:10.1186/s11671-019-3046-3

8. Ferrer-Anglada N, Gomis V, El-Hachemi Z, et al. Carbon nanotube based composites for electronic applications: CNT-conducting polymers, CNT-Cu. Phys Status Solidi A. 2006;203(6):1082-1087. doi:10.1002/pssa.200566188

9. Saeb MR, Najafi F, Bakhshandeh E, et al. Highly curable epoxy/ MWCNTs nanocomposites: an effective approach to functionalization of carbon nanotubes. Chem Eng J. 2015;259:117-125. doi:10.1016/j.cej.2014.07.116

10. Chandrasekhar P. CNT Applications in Drug and Biomolecule Delivery, in Conducting Polymers, Fundamentals and Applications. Springer; 2018:61-64.

11. Pastorin G, Wu W, Wieckowski S, et al. Double functionalisation of carbon nanotubes for multimodal drug delivery. Chem Commun. 2006;11(11):1182-1184. doi:10.1039/b516309a

12. Prato M, Kostarelos K, Bianco A. Functionalized carbon nanotubes in drug design and discovery. Acc Chem Res. 2008;41(1):60-68. doi:10.1021/ar700089b

13. Skandani AA, Al-Haik M. Reciprocal effects of the chirality and the surface functionalization on the drug delivery permissibility of carbon nanotubes. Soft Matter. 2013;9(48):11645-11649. doi:10.1039/ C3SM52126E 
14. Dehaghani MZ, Yousefi F, Seidi F, et al. Encapsulation of an anticancer drug Isatin inside a host nano-vehicle SWCNT: a molecular dynamics simulation. Sci Rep. 2021;11(1):1-10. doi:10.1038/s41598021-98222-2

15. Bernkop-Schnürch A, Bratengeyer I, Valenta C. Development and in vitro evaluation of a drug delivery system protecting from trypsinic degradation. Int J Pharm. 1997;157(1):17-25. doi:10.1016/S03785173(97)00198-1

16. Smith AW. Biofilms and antibiotic therapy: is there a role for combating bacterial resistance by the use of novel drug delivery systems? Adv Drug Deliv Rev. 2005;57(10):1539-1550. doi:10.1016/j. addr.2005.04.007

17. Zarrintaj P, Ramsey JD, Samadi A, et al. Poloxamer: a versatile triblock copolymer for biomedical applications. Acta Biomater. 2020;110:37-67. doi:10.1016/j.actbio.2020.04.028

18. Ramos MADS, Da Silva P, Spósito L, et al. Nanotechnology-based drug delivery systems for control of microbial biofilms: a review. Int J Nanomedicine. 2018;13:1179. doi:10.2147/IJN.S146195

19. Ménard-Moyon C, Venturelli E, Fabbro C, et al. The alluring potential of functionalized carbon nanotubes in drug discovery. Expert Opin Drug Discov. 2010;5(7):691-707. doi:10.1517/17460441.2010.490552

20. Sekar G, Kandiyil ST, Sivakumar A, et al. Binding studies of hydroxylated Multi-Walled Carbon Nanotubes to hemoglobin, gamma globulin and transferrin. J Photochem Photobiol B. 2015;153:222-232. doi:10.1016/j.jphotobiol.2015.09.023

21. Chen Z, Pierre D, He H, et al. Adsorption behavior of epirubicin hydrochloride on carboxylated carbon nanotubes. Int J Pharm. 2011;405(1-2):153-161. doi:10.1016/j.ijpharm.2010.11.034

22. Zardini HZ, Amiri A, Shanbedi M, et al. Enhanced antibacterial activity of amino acids-functionalized multi walled carbon nanotubes by a simple method. Colloids Surf B Biointerfaces. 2012;92:196-202. doi:10.1016/j.colsurfb.2011.11.045

23. Liu P. Modification strategies for carbon nanotubes as a drug delivery system. Ind Eng Chem Res. 2013;52(38):13517-13527. doi:10.1021/ ie402360f

24. Tan JM, Arulselvan P, Fakurazi S, et al. A review on characterizations and biocompatibility of functionalized carbon nanotubes in drug delivery design. J Nanomater. 2014;2014:1-20. doi:10.1155/2014/ 917024

25. Liu Z, Liu Y, Peng D. Hydroxylation of multi-walled carbon nanotubes reduces their cytotoxicity by limiting the activation of mitochondrial mediated apoptotic pathway. J Mater Sci Mater Med. 2014;25(4):1033-1044.

26. Maleki R, Afrouzi HH, Hosseini M, et al. pH-sensitive loading/ releasing of doxorubicin using single-walled carbon nanotube and multi-walled carbon nanotube: a molecular dynamics study. Comput Methods Programs Biomed. 2020;186:105210. doi:10.1016/j. cmpb.2019.105210

27. Moradnia H, Raissi H, Shahabi M. The performance of the singlewalled carbon nanotube covalently modified with polyethylene glycol to delivery of Gemcitabine anticancer drug in the aqueous environment. J Biomol Struct Dyn. 2020;39:881-888.

28. Kamel M, Raissi H, Morsali A, et al. Assessment of the adsorption mechanism of Flutamide anticancer drug on the functionalized single-walled carbon nanotube surface as a drug delivery vehicle: an alternative theoretical approach based on DFT and MD. Appl Surf Sci. 2018;434:492-503. doi:10.1016/j.apsusc.2017.10.165

29. Dehneshin N, Raissi H, Hasanzade Z, et al. Using molecular dynamics simulation to explore the binding of the three potent anticancer drugs sorafenib, streptozotocin, and sunitinib to functionalized carbon nanotubes. J Mol Model. 2019;25(6):159. doi:10.1007/ s00894-019-4024-5

30. Hashemzadeh H, Raissi $H$. The functionalization of carbon nanotubes to enhance the efficacy of the anticancer drug paclitaxel: a molecular dynamics simulation study. J Mol Model. 2017;23(8):1-10. doi:10.1007/s00894-017-3391-z
31. Molaei F, Zarghami dehaghani M, Salmankhani A, et al. Applying molecular dynamics simulation to take the fracture fingerprint of polycrystalline $\mathrm{SiC}$ nanosheets. Comput Mater Sci. 2021;200:110770. doi:10.1016/j.commatsci.2021.110770

32. Fooladpanjeh S, Yousefi F, Molaei F, et al. Thermal conductivity of random polycrystalline $\mathrm{BC} 3$ nanosheets: a step towards realistic simulation of 2D structures. J Mol Graph Model. 2021;107:107977. doi:10.1016/j.jmgm.2021.107977

33. Dehaghani MZ, Esmaeili Safa M, Yousefi F, et al. Fracture behavior of SiGe nanosheets: mechanics of monocrystalline vs. polycrystalline structure. Eng Fract Mech. 2021;251:107782. doi:10.1016/j. engfracmech.2021.107782

34. Bagheri B, Zarghami dehaghani M, Esmaeili Safa M, et al. Fracture fingerprint of polycrystalline $\mathrm{C} 3 \mathrm{~N}$ nanosheets: theoretical basis. $\mathrm{J} \mathrm{Mol}$ Graph Model. 2021;106:107899. doi:10.1016/j.jmgm.2021.107899

35. Salmankhani A, Karami Z, Hamed Mashhadzadeh A, et al. A theoretical scenario for the mechanical failure of boron carbide nanotubes. Comput Mater Sci. 2021;186:110022. doi:10.1016/j. commatsci.2020.110022

36. Dehaghani MZ, Salmankhani A, Hamed Mashhadzadeh A, et al. Fracture mechanics of polycrystalline beryllium oxide nanosheets: a theoretical basis. Eng Fract Mech. 2021;244:107552. doi:10.1016/j. engfracmech.2021.107552

37. Bagheri B, Zarghami Dehaghani M, Karami Z, et al. Correlation between surface topological defects and fracture mechanism of $\gamma$ graphyne-like boron nitride nanosheets. Comput Mater Sci. 2021;188:110152. doi:10.1016/j.commatsci.2020.110152

38. Dehaghani MZ, Hamed Mashhadzadeh A, Salmankhani A, et al. Fracture toughness and crack propagation behavior of nanoscale beryllium oxide graphene-like structures: a molecular dynamics simulation analysis. Eng Fract Mech. 2020;235:107194. doi:10.1016/j.engfracmech.2020.107194

39. Zarghami dehaghani M, Yousefi F, Sajadi SM, et al. Theoretical encapsulation of fluorouracil (5-FU) anti-cancer chemotherapy drug into carbon nanotubes (CNT) and boron nitride nanotubes (BNNT). Molecules. 2021;26(16):4920. doi:10.3390/molecules26164920

40. Dehaghani MZ, Yousefi F, Bagheri B, et al. $\alpha$-helical antimicrobial peptide encapsulation and release from boron nitride nanotubes: a computational study. Int $J$ Nanomedicine. 2021;16:4277. doi:10.2147/IJN.S313855

41. Dehaghani MZ, Bagheri B, Yousefi F, et al. Boron nitride nanotube as an antimicrobial peptide carrier: a theoretical insight. Int $J$ Nanomedicine. 2021;16:1837. doi:10.2147/IJN.S298699

42. Zarghami Dehaghani M, Bagheri B, Nasiriasayesh A, et al. Insight into the self-insertion of a protein inside the boron nitride nanotube. $A C S$ Omega. 2020;5(49):32051-32058. doi:10.1021/acsomega.0c05080

43. Zhu S, Aumelas A, Gao B. Convergent evolution-guided design of antimicrobial peptides derived from influenza A virus hemagglutinin. J Med Chem. 2011;54(4):1091-1095. doi:10.1021/jm1010463

44. Plimpton S. Fast parallel algorithms for short-range molecular dynamics. $J$ Comput Phys. 1995;117(1):1-19. doi:10.1006/jcph.1995.1039

45. Humphrey W, Dalke A, Schulten K. VMD: visual molecular dynamics. J Mol Graph. 1996;14(1):33-38. doi:10.1016/0263-7855 (96)00018-5

46. Park S, Schulten K. Calculating potentials of mean force from steered molecular dynamics simulations. J Chem Phys. 2004;120(13):59465961. doi:10.1063/1.1651473

47. Tersoff J. New empirical approach for the structure and energy of covalent systems. Phys Rev B. 1988;37(12):6991-7000. doi:10.1103/ PhysRevB.37.6991

48. Hirschfelder JO, Curtiss CF, Bird RB. Molecular Theory of Gases and Liquids. Vol. 165. New York: Wiley; 1964.

49. Kang Y, Liu Y-C, Wang Q, et al. On the spontaneous encapsulation of proteins in carbon nanotubes. Biomaterials. 2009;30(14):2807-2815. doi:10.1016/j.biomaterials.2009.01.024 
50. Feller SE, Zhang Y, Pastor RW, et al. Constant pressure molecular dynamics simulation: the Langevin piston method. J Chem Phys. 1995;103(11):4613-4621. doi:10.1063/1.470648

51. Mousavi SZ, Amjad-Iranagh S, Nademi Y, et al. Carbon nanotubeencapsulated drug penetration through the cell membrane: an investigation based on steered molecular dynamics simulation. $J \mathrm{Membr}$ Biol. 2013;246(9):697-704. doi:10.1007/s00232-013-9587-y

52. Gao H, Kong Y, Cui D, et al. Spontaneous insertion of DNA oligonucleotides into carbon nanotubes. Nano Lett. 2003;3(4):471-473. doi:10.1021/n1025967a

53. Kang Y, Wang Q, Liu YC, et al. Dynamic mechanism of collagenlike peptide encapsulated into carbon nanotubes. J Phys Chem B. 2008;112(15):4801-4807. doi:10.1021/jp711392g

54. Maleki R, Afrouzi HH, Hosseini M, et al. Molecular dynamics simulation of Doxorubicin loading with $\mathrm{N}$-isopropyl acrylamide carbon nanotube in a drug delivery system. Comput Methods Programs Biomed. 2020;184:105303. doi:10.1016/j.cmpb.2019.105303

55. Veclani D, Melchior A. Adsorption of ciprofloxacin on carbon nanotubes: insights from molecular dynamics simulations. J Mol Liq. 2020;298:111977. doi:10.1016/j.molliq.2019.111977
56. Ghadri Z, Raissi H, Shahabi M, et al. Molecular dynamics simulation study of Glycine tip-functionalisation of single-walled carbon nanotubes as emerging nanovectors for the delivery of anticancer drugs. Mol Simul. 2020;46(2):111-120. doi:10.1080/08927022.2019.1679363

57. Zhang D, Gullingsrud J, McCammon JA. Potentials of mean force for acetylcholine unbinding from the alpha7 nicotinic acetylcholine receptor ligand-binding domain. $J$ Am Chem Soc. 2006;128 (9):3019-3026. doi:10.1021/ja057292u

58. Zhang L, Peng G, Li J, et al. Molecular dynamics study on the configuration and arrangement of doxorubicin in carbon nanotubes. J Mol Liq. 2018;262:295-301. doi:10.1016/j.molliq.2018.04.097

59. Shen JW, Tang T, Wei XH, et al. On the loading mechanism of ssDNA into carbon nanotubes. RSC Adv. 2015;5(70):56896-56903. doi:10.1039/C5RA01941A

60. Shen J-W, Wu T, Wang Q, et al. Induced stepwise conformational change of human serum albumin on carbon nanotube surfaces. Biomaterials. 2008;29(28):3847-3855. doi:10.1016/j.biomaterials.2008.06.013

\section{Publish your work in this journal}

The International Journal of Nanomedicine is an international, peerreviewed journal focusing on the application of nanotechnology in diagnostics, therapeutics, and drug delivery systems throughout the biomedical field. This journal is indexed on PubMed Central, MedLine, CAS, SciSearch ${ }^{\mathbb{R}}$, Current Contents ${ }^{\mathbb{R}} /$ Clinical Medicine, $^{-}$
Journal Citation Reports/Science Edition, EMBase, Scopus and the Elsevier Bibliographic databases. The manuscript management system is completely online and includes a very quick and fair peer-review system, which is all easy to use. Visit http://www.dovepress.com/ testimonials.php to read real quotes from published authors. 